\title{
Comparação do Treinamento Físico de Quatro e Oito Semanas sobre Atividade da Cadeia Transportadora de Elétrons e Marcadores de Estresse Oxidativo em Fígado de Camundongos
}

\section{Comparison Between Four- And Eight- Week Physical Trainings on the Mitochondrial Respiratory Chain Enzyme Activities and Oxidative Stress Markers in Liver of Mice}

Luciano A. Silva'

Merieli M. Ronsani'

Priscila S. Souza'

Bruna J. Severino

Daiane B. Fraga²

Emilio I. Streck, PhD²

Ricardo A. Pinho, PhD ${ }^{1}$

1. Laboratório de Fisiologia e Bioquímica do Exercício/UNESC. 2. Laboratório de Fisiopatologia Experimental/UNESC

Endereço para correspondência: Universidade do Extremo Sul Catarinense - Unesc

Laboratório de Fisiologia e

Bioquímica do Exercício/Bloco da

Saúde

Av. Universitária, 1.105 - Bairro

Universitário

88806-000 - Criciúma, SC - Brasil

E-mail: luciano_acordi@yahoo.com.br

\begin{abstract}
RESUMO
O presente estudo investigou o efeito de quatro e oito semanas de treinamento físico sobre a atividade dos complexos da cadeia transportadora de elétrons (CTE) e os marcadores de estresse oxidativo em fígado de camundongos. Vinte e um camundongos (CF1, 30-35g) foram distribuídos nos seguintes grupos: não treinado (NT); treinado quatro semanas (T4); treinado oito semanas (T8). Quarenta e oito horas após a última sessão de treinamento os animais foram mortos por decapitação e o fígado foi retirado e estocado em $-70^{\circ} \mathrm{C}$ para posterior análise. Atividade da succinato desidrogenase (SDH), dos complexos I,II,III e IV da CTE, carbonilação de proteína, conteúdo total de tióis e a atividade da superóxido dismutase foram mensurados. Os resultados demonstram que apenas oito semanas de treinamento aumentam a atividade da SDH, dos quatro complexos da CTE, da superóxido dismutase, e o conteúdo total de tióis em relação ao grupo não treinado. Houve ainda diminuição na carbonilação de proteína no respectivo grupo em relação ao NT. Em conclusão, são necessárias oito semanas de treinamento para que ocorram aumento no funcionamento mitocondrial e melhora nos marcadores de estresse oxidativo em fígado de camundongos.
\end{abstract}

Palavras-chave: treinamento físico, cadeia transportadora de elétrons, estresse oxidativo

\begin{abstract}
The present study investigated mitochondrial adaptations and oxidative stress markers after four and eight weeks of running training in liver of mice. Twenty-one male mice (CF1, 30-35g) were distributed into the following groups ( $n=7$ ): untrained (UT); trained - four weeks (T4); trained - eight weeks (T8). Forty-eight hours after the last training session the animals were killed by decapitation and livers were removed and stored at $-70^{\circ} \mathrm{C}$. Succinate dehydrogenase (SDH), complexes I, II, II-III and IV, protein carbonyls (PC), total thiol content and superoxide dismutase activity were measured. The results show that endurance training (8-wk) increases the SDH activity and complexes (I, II, III, IV), superoxide dismutase and total thiol content in liver when compared to untrained animals. Decrease in protein carbonylation in the respective group in relation to UT was also observed. It could be concluded that eight weeks of running training are necessary for mitochondrial respiratory chain enzyme activities increase and improvement in oxidative stress markers in liver of mice.
\end{abstract}

Keywords: physical training, electron transport chain, oxidative stress

\section{INTRODUÇÃO}

Estudos têm reportado que o exercício físico aumenta a produção das espécies reativas de oxigênio (ERO) e, consequentemente, estresse oxidativo em diversos órgãos e tecidos ${ }^{(1,2)}$. Tem sido sugerido que a elevação do consumo de oxigênio induzida pelos exercícios aumenta a atividade mitocondrial, gerando altas concentrações de $E R O^{(3)}$. $O$ estresse oxidativo (EO) altera a integridade de constituintes celulares e compromete todo o funcionamento celular ${ }^{(1)}$. Células hepáticas precisam de energia para realizar diversas funções. A alta taxa metabólica do fígado (200kcal $/ \mathrm{kg} / \mathrm{tecido} / \mathrm{dia}$ ) está diretamente associada a um alto fluxo de elétrons na cadeia respiratória mitocondrial(4). Entretanto, parte desses elétrons é desviado, produzindo ERO adicionais.
Especificadamente no fígado, diversos autores demonstram que esse órgão sofre aumento de estresse oxidativo após o exercício físico ${ }^{(1,4)}$. Por outro lado, outros estudos têm reportado que o treinamento regular tem sido eficaz, na diminuição do estresse oxidativo provocado por diversas doenças( ${ }^{(5,6)}$. Entretanto a comparação dos efeitos do treinamento de quatro e oito semanas sobre a atividade mitocondrial e marcadores de estresse oxidativo em fígados de camundongos permanece obscura.

A hipótese deste estudo pressupõe que são necessárias no mínimo oito semanas de treinamento físico regular para que ocorram adaptações na cadeia transportadora de elétrons que provoquem aumento nas defesas antioxidantes e diminuição no dano oxidativo. Sendo assim, 
o presente estudo tem como objetivo investigar o efeito de quatro e oito semanas de treinamento físico sobre a atividade dos complexos da cadeia transportadora de elétrons e os marcadores de estresse oxidativo em fígado de camundongos.

\section{METODOLOGIA}

Todos os procedimentos foram realizados de acordo com Guiding Principles in the care and uses of animals ${ }^{(7)}$ e aprovados pelo comitê de ética da Universidade do Extremo Sul Catarinense-SC, Brasil.

\section{Amostra}

Vinte e um camundongos CF1 machos com 60 dias, pesando entre 30 e 40g, foram divididos em três grupos $(n=7)$. Grupo 1: não treinados (NT); grupo 2: treinamento quatro semanas (T4); grupo 3: treinamento oito semanas (T8). Os animais ficaram alocados em três gaiolas no biotério da Universidade local, onde tiveram acesso livre a água e comida, com temperatura ambiente de 23 graus controlada com termostato e ciclo de claro e escuro de 12h.

\section{Protocolos de treinamento}

Todos os animais foram adaptados durante uma semana, uma vez ao dia, cinco dias por semana com velocidade $\left(10 \mathrm{~m} \cdot \mathrm{min}^{-1}\right)$ e tempo total da sessão de dez minutos. Após o período de adaptação os grupos 2 e 3 foram treinados de acordo com a tabela 1.

\section{Sacrifício dos animais}

Quarenta e oito horas após a última seção de treinamento os ratos foram mortos por guilhotina e o fígado foi retirado cirurgicamente para posterior análise.

\section{Ensaios bioquímicos}

\section{Preparação de tecido e homogeneizado}

Após o sacrifício o fígado foi rapidamente removido e separado em duas porções. A primeira porção foi homogeneizada imediatamente (1:10w/v) em tampão SETH, pH 7,4 (250mM sacarose, 2mM EDTA, 10mM Trizma base, 50IU/mL heparina), centrifugado a 800 X g por 10 min e o sobrenadante foi estocado a $-70^{\circ} \mathrm{C}$ para a análise das atividades dos complexos. A segunda porção de tecido foi congelada imediatamente para análise dos marcadores de estresse oxidativo.

\section{Succcinato desidrogenase}

A atividade da SDH foi verificada utilizado o sistema 2,6-DCIP em $600 \mathrm{~nm}$ na presença de metassulfato de fenazina, segundo o método de Fischer et al. ${ }^{(8)}$.

\section{Atividade do complexo I}

A atividade da NADH desidrogenase foi avaliada pelo método descrito por Cassina e Radi( ${ }^{(9)}$ pela taxa de $\mathrm{NADH}$ dependente da redução do ferricianeto a $420 \mathrm{~nm}$.

Tabela 1. Protocolo de treinamento de quatro e oito semanas.

\begin{tabular}{c|c|c}
\hline Semanas & Velocidade $\left(\mathbf{m} \cdot \mathbf{m i n}^{-1}\right)$ & Duração (minutos) \\
\hline 1 & 13,0 & 45 \\
\hline 2 & 13,0 & 45 \\
\hline 3 & 13,0 & 45 \\
\hline 4 & 13,0 & 45 \\
\hline 5 & 13,0 & 45 \\
\hline 6 & 13,0 & 45 \\
\hline 7 & 13,0 & 45 \\
\hline 8 & 13,0 & 45 \\
\hline
\end{tabular}

\section{Atividade do complexo II}

A atividade enzimática foi medida pelo método descrito por Fischer et al. ${ }^{(8)}$, onde a diminuição da absorbância do 2,6-DCIP em 600nm é usada para o cálculo da atividade do complexo II.

\section{Atividade do complexo II-III}

A atividade do citocromo c oxiredutase (complexo II-III) foi determinada de acordo com Fischer et al. ${ }^{(8)}$, onde foi medida pela redução do citocromo c para succinato.

\section{Atividade do complexo IV}

A atividade do complexo IV foi determinada de acordo com Rustin et al. ${ }^{(10)}$, e é calculada pela diminuição da absorbância causada pela oxidação do citocromo c reduzido, medido em 550nm.

\section{Carbonilação de proteínas}

Os danos oxidativos em proteínas foram calculados pela determinação de grupos carbonils baseados na reação com dinitrofenilhidrazina, como previamente descrito. O conteúdo de carbonil foi determinado espectrofotometricamente em 370nm usando um coeficiente 22.0000 molar $^{-1(11)}$

\section{Tióis totais}

Foi determinada numa reação dos grupos tióis com 5,5 ditióbis (2 ácido nitro-benzoico) (DTNB), gerando um derivado de coloração amarela e lido espectrofotometricamente a 412nm(12). Essa técnica tem por objetivo avaliar o grupo SH (sulfidrila) não oxidado.

\section{Superóxido dismutase (SOD)}

A atividade enzimática da SOD foi determinada pela inibição da auto-oxidação da adrenalina medida espectrofotometricamente (480nn) segundo Bannister e Calabrese ${ }^{(13)}$.

\section{Determinação da proteína}

A quantidade de proteínas nos ensaios da carbonilação de proteínas e no conteúdo de tíois totais foi mensurada usando a técnica de Lowry et al.14).

\section{Tratamento estatístico}

Os dados foram expressos em média e erro padrão médio e analisados estatisticamente pela análise de variância (ANOVA) one-way, seguida pelo teste post hoc de Bonferroni. O nível de significância estabelecido para o teste estatístico é de $p<0,05$. Foi utilizado o SPSS (Statistical Package for the Social Sciences) versão 15.0 como pacote estatístico.

\section{Reagentes}

Acido tricloroacético, ácido clorídrico, dinitrofenilhidrazina (CP), ácido ditionitrobenzoico, fosfato de potássio, ácido etilenodiamino tetra-acético (TT), succinato de sódio, azida sódica, rotenona, citocromo, sacarose, trizma base, heparina, etanol absoluto, metassulfato de fenazina, hidróxido de sódio (CTE), albumina, carbonato de sódio, sulfato de cobre, tartarato (PT).

\section{RESULTADOS}

\section{Enzima do ciclo de Krebs}

\section{Succinato desidrogenase (SDH)}

Os resultados demonstraram aumento significativo na atividade da SDH no grupo T8 (7,42 \pm 2,27nmol/mg proteina) em comparação com o NT (2,23 $\pm 0,7 \mathrm{nmol} / \mathrm{mg}$ proteina) (Tabela 2$)$. 
Tabela 2. Atividade das enzimas da cadeia respiratória mitocondrial no fígado de camundongos após 48 horas da ultima sessão do programa de treinamento. Os animais foram distribuídos em três grupos $(n=7)$ : não treinado (NT), quatro semanas de treinamento (T4), oito semanas de treinamento (T8). Os valores são apresentados em média \pm EPM e os resultados são expressos em nmol/min/mg proteína. A diferença significativa foi encontrada em relação ao grupo não treinado(*) com o p $<0,05$.

\begin{tabular}{c|c|c|c}
\hline & $\begin{array}{c}\text { (NT) } \\
\text { nmol/min mg } \\
\text { proteína }\end{array}$ & $\begin{array}{c}\text { (T4) } \\
\text { nmol/min mg } \\
\text { proteína }\end{array}$ & $\begin{array}{c}\text { (T8) } \\
\text { nmol/min } \mathbf{~ m g ~} \\
\text { proteína }\end{array}$ \\
\hline SDH & $2,23 \pm 0,7$ & $4,48 \pm 2,4$ & $7,42 \pm 2,27^{*}$ \\
\hline Complexo I & $20,8 \pm 41$ & $39,2 \pm 9,7$ & $99,4 \pm 13^{*}$ \\
\hline Complexo II & $1,1 \pm 0,2$ & $1,5 \pm 0,2$ & $4,3 \pm 0,5^{*}$ \\
\hline Complexo II-III & $1,0 \pm 0,1$ & $1,5 \pm 0,2$ & $3,9 \pm 0,2^{*}$ \\
\hline Complexo IV & $70,4 \pm 10$ & $118 \pm 21$ & $200 \pm 27^{*}$ \\
\hline
\end{tabular}

\section{Atividade das enzimas da cadeia transportadora de elétrons Complexo I}

Ocorreu aumento significativo na atividade NADH desidrogenase no grupo T8 $(99,4 \pm 32,5 \mathrm{nmol} / \mathrm{min} / \mathrm{mg}$ ) quando comparado com não treinado NT (20,8 $\pm 11,7 \mathrm{nmol} / \mathrm{min} / \mathrm{mg})$ (Tabela 2).

\section{Complexo II}

Houve aumento significativo na atividade do complexo II no grupo T8 (4,3 $\pm 1,4 \mathrm{nmol} / \mathrm{min} / \mathrm{mg})$ em relação ao grupo não treinado(1,1 \pm 0,3nmol/min/mg) (Tabela 2).

\section{Complexo II-III}

A atividade do citocromo c oxirredutase aumentou significativamente no grupo T8 $(3,65 \pm 0,7 \mathrm{nmol} / \mathrm{min} / \mathrm{mg})$ em comparação com o não NT $(0,89 \pm 0,2 \mathrm{nmol} / \mathrm{min} / \mathrm{mg})$ (Tabela 2).

\section{Complexo IV}

A atividade do citocromo c aumentou significativamente no grupo T8 (70,4 \pm 22,7nmol/min/mg) em comparação com o NT (200 土 $61,5 \mathrm{nmol} / \mathrm{min} / \mathrm{mg}$ ) (Tabela 2).

\section{Conteúdo de proteínas oxidadas e não oxidadas \\ Carbonilação de proteína}

Os danos em proteínas diminuíram significativamente no grupo T8 (2,24 \pm 0,7nmol/mg proteína) em comparação com o não NT (4,46 $\pm 0,6 \mathrm{nmol} / \mathrm{mg}$ proteína) (Figura 1).

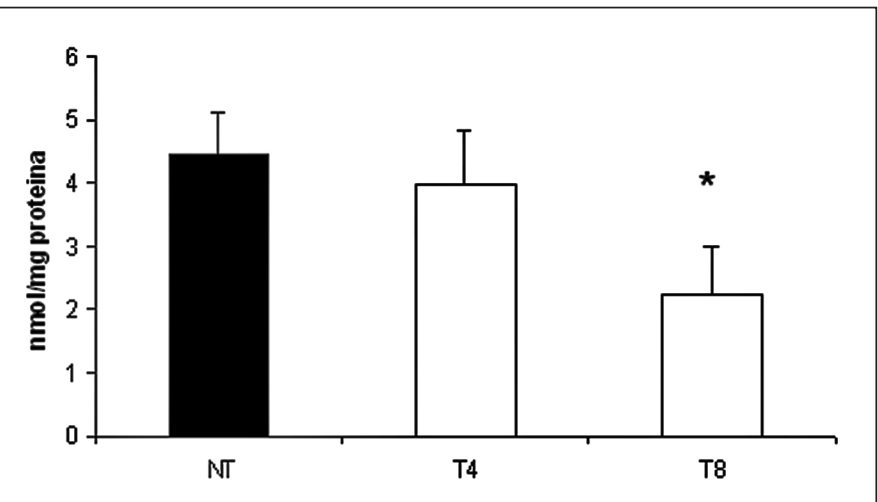

Figura 1. Carbonilação de proteína no fígado de camundongos após 48 horas da ultima sessão do programa de treinamento. Os animais foram distribuídos em três grupos ( $n=7)$ : não treinado (NT), quatro semanas de treinamento (T4), oito semanas de treinamento (T8). Os valores são apresentados em média \pm EPM e os resultados são expressos em nmol/mg proteína. A diferença significativa foi encontrada em relação ao grupo não treinado $\left(^{*}\right)$ com o $p<0,05$.

\section{Tióis totais}

O conteúdo de tióis totais aumentou significativamente no grupo T8 (7,5 \pm 0,8nmol/mg proteína) em comparação com o NT (5,15 \pm 07nmolTNB/mg proteína) (Figura 2).

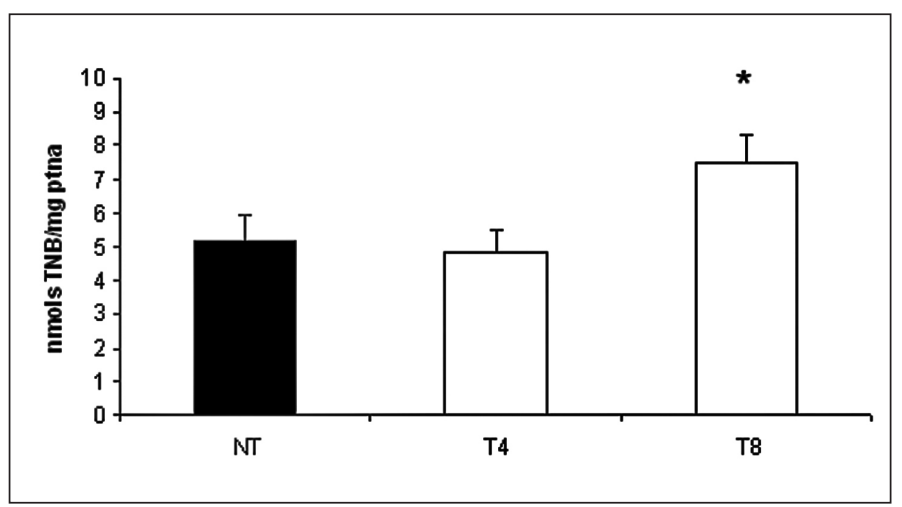

Figura 2. Conteúdo total de tióis no fígado de camundongos após 48 horas da ultima sessão do programa de treinamento. Os animais foram distribuídos em três grupos $(n=7)$ : não treinado (NT), quatro semanas de treinamento (T4), oito semanas de treinamento (T8). Os valores são apresentados em média \pm EPM e os resultados são expressos em nmol/TNB/mg proteína. A diferença significativa foi encontrada em relação ao grupo não treinado(*) com o $p<0,05$.

\section{Atividade antioxidante da enzimática}

\section{Superóxido dismutase (SOD)}

A atividade da SOD aumentou significativamente no grupo T8 (0,83 \pm 0,08U/mg de proteína) em comparação com o NT (0,47 $\pm 0,04 \mathrm{U} / \mathrm{mg}$ de proteína) (Figura 3).

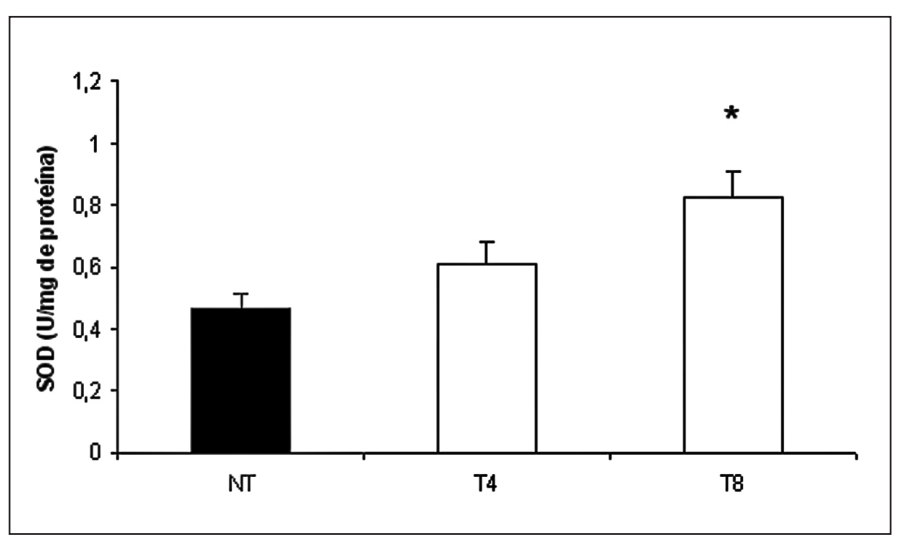

Figura 3. Atividade da superóxido dismutase em fígado de camundongos após 48 horas da ultima sessão do programa de treinamento. Os animais foram distribuídos em três grupos ( $n=7)$ : não treinado (NT), quatro semanas de treinamento (T4), oito semanas de treinamento (T8). Os valores são apresentados em média \pm EPM e os resultados são expressos em $\mathrm{U} / \mathrm{mg}$ proteína. A diferença significativa foi encontrada em relação ao grupo não treinado(*) $\operatorname{com} o p<0,05$.

\section{DISCUSSÃO}

O presente estudo demonstrou que quatro semanas de treinamento físico não são suficientes para aumentar a atividade dos complexos da CTE e reduzir parâmetros de estresse oxidativo em fígado de ratos. São necessárias oito semanas de treinamento para que ocorra aumento na atividade CTE e diminuição de EO.

Disfunções mitocondriais são caracterizadas pela redução do controle respiratório, diminuição no potencial de membrana e desacoplamento mitocondrial(15). Essas alterações são observadas no envelhecimento e em situações patológicas ${ }^{(5,15)}$. Prejuízos na atividade 
do complexo IV, por exemplo, estão relacionados com a doença de Alzheimer ${ }^{(16)}$ e do complexo I, com Parkinson ${ }^{(17)}$. No fígado especificadamente, a esteatose reduz a atividade da CTE, diminuindo os níveis de ATP, e desenvolve estresse oxidativo ${ }^{(18)}$. Demonstramos que aumentar a atividade da CTE e reduzir o estresse oxidativo é possível através do treinamento. Acreditamos que no futuro isso pode ser útil na prevenção e combate de diversas doenças, inclusive as de ordem hepática.

Durante o exercício físico ocorre aumento significativo na produção de ATP, o qual está associado a aumento do fluxo mitocondrial|(3). Nossos achados demonstram que o treinamento físico regular de oito semanas aumenta a atividade da SDH e dos quatro complexos da CTE. Diversos estudos têm usado a SDH como marcador da capacidade oxidativa ${ }^{(19,20)}$. Bagby et al. ${ }^{(20)}$ demonstraram aumento da atividade dessa enzima, depois de sete e 16 semanas de exercício voluntário (rodas de correr) em ratos. Resultados similares são apontados por outros autores ${ }^{(21)}$, no que diz respeito a mudanças enzimáticas no funcionamento mitocondrial após o treinamento. Dados do nosso grupo mostram que a atividade da SDH e dos quatro complexos da CTE são elevados após oito semanas de treinamento em músculo esquelético ${ }^{(22)}$. Contudo, os resultados do presente estudo demonstram que o fígado responde de maneira similar. Nakamoto et al. ${ }^{(23)}$ confirmaram que o exercício regular promove adaptações no funcionamento mitocondrial no fígado, reduzindo o dano oxidativo. O treinamento aeróbico está associado ao aumento da atividade das enzimas da CTE e concomitantemente ao aumento da concentração de proteínas ${ }^{(24)}$. É possível que o aumento da atividade dos complexos CTE provocado pelo exercício seja suficiente para reduzir os marcadores de estresse oxidativo.

Como marcadores de estresse oxidativo foi avaliado o conteúdo de proteínas oxidadas (carbonil), não oxidadas (tióis totais) e atividade antioxidante da superóxido dismutase (SOD). Nossos achados apontam que oito semanas de treinamento reduzem o conteúdo de proteínas oxidadas, aumentam os níveis de tióis totais e a atividade enzimática da SOD. Geralmente, tem sido reportado que o exercício regular resulta no aumento da atividade das enzimas antioxidantes, aumentando a

\section{REFERÊNCIAS}

1. Navarro-Arevalo A, Sanchez-del-Pino MJ. Age and exercise-related changes in lipid peroxidation and superoxide dismutase activity in liver and soleus muscle tissues of rats. Mech Ageing Dev 1998;104:91-102.

2. Silva LA, Silveira PC, Pinho CA, Tuon T, Dal Pizzol F, Pinho RA. N-acetylcysteine supplementation and oxidative damage and inflammatory response after eccentric exercise. Int J Sport Nutr Exerc Metab 2008;18:379-88.

3. Servais S, Couturier K, Koubi H, Rouanet JL, Desplanches D, Sornay-Mayet MH, et al._Effect of voluntary exercise on $\mathrm{H} 2 \mathrm{O} 2$ release by subsarcolemmal and intermyofibrillar emitochondria. Free Radic Biol Med 2003;35:24-32.

4. Ogonovszky H, Sasvári M, Dosek A, Berkes I, Kaneko T, Tahara S, et al. The effects of moderate, strenuous, and overtraining on oxidative stress markers and DNA repair in rat liver. Can J Appl Physiol 2005;30:186-95.

5. Pinho RA, Chiesa D, Mezzomo KM, Andrades ME, Bonatto F, Gelain D, et al. Oxidative stress in chronic obstructive pulmonary disease patients submitted to a rehabilitation program. Respir Med 2007;101:1830-5.

6. Aguiar AS Jr, Tuon T, Albuquerque MM, Rocha GS, Speck AE, Araújo JC, et al. The exercise redox paradigm in the Down's syndrome: improvements in motor function and increases in blood oxidative status in young adults. J Neural Transm 2008;115:1643-50.

7. Olert E, Cross B, Mcwillians A. Guide to care and use of experimental animals. 2nd ed. Canadian Council on Animal, Ottawa.

8. Fischer JC, Ruitenbeek W, Berden JA, Trijbels JM, Veerkamp JH, Stadhouders AM, et al. Differential investigation of the capacity of succinate oxidation in human skeletal muscle. Clin Chim Acta 1985;153:23-6.

9. Cassina A, Radi R. Differential inhibitory action of nitric oxide and peroxynitrite on mitochondrial electron transport. Arch Biochem Biophys 1996;328:309-16.

10. Rustin P, Chretien D, Bourgeron T, Gerard B, Rotig A, Saudubray JM, et al. Biochemical and molecular investigations in respiratory chain deficiencies. Clin Chim Acta 1994;228:35-51.

11. Levine RL, Garland D, Oliver CN, Amici A, Climent I, Lenz AG, et al. Determination of carbonyl content in oxidatively modified proteins. Meth Enzymol 1990;186:464-78.

12. Aksenov MY, Markesberya WR. Changes in thiol content and expression of glutathione redox system genes in the hippocampus and cerebellum in Alzheimer's disease. Neurosci Lett 2001;302:141-5.

13. Bannister JV, Calabrese L. Assay for SOD. Meth Biochem. 1987;32:279-312.

14. Lowry OH, Rosebrough NJ, Farr AL, Randall RJ. Protein measurement with the Folin phenol reagent. J Biol Chem 1951;193:265-7. proteção contra $\mathrm{ERO}^{(25,26)}$. Entretanto, alguns estudos têm apontado que o treinamento físico não tem nenhum efeito sobre as enzimas antioxidantes no fígado ${ }^{(4)}$. Corroborando nossos resultados, Navarro et al. ${ }^{(26)}$ demonstraram que o treinamento aeróbico aumenta a atividade da SOD no fígado. Acreditamos que as discrepâncias encontradas nos resultados citados podem estar relacionadas com os diferentes protocolos de exercício (intensidade, duração, frequência e tipo).

Todavia, Venditti et al.(27) demonstraram que o treinamento de aeróbio reduziu a produção de ERO mitocondrial. Powers et al. ${ }^{(28)}$ encontraram benefícios no aumento nas enzimas antioxidantes após o treinamento de 10 semanas. Nakamoto et al.(23) demonstraram que o exercício regular reduz o estresse oxidativo em fígado de ratos. Uma das hipóteses, de acordo com Nakamoto et al. ${ }^{(29)}$ para explicar a redução de estresse oxidativo no fígado é que exercício ativa fatores de transcrição que se ligam ao DNA mitocondrial e nuclear, causando adaptações que protegem as células contra o ataque das ERO. Segundo Pinho et al. ${ }^{(5)}$, outro fator que poderia explicar esse fenômeno é a exposição crônica do tecido às $E R O$, induzida pelo treinamento, tornando o órgão mais resistente ao estresse oxidativo. Uma terceira hipótese sustentada por Gonzalez et al. ${ }^{(29)}$ é o aumento das proteínas da choque (HSP) induzidas pelo exercício. Essas proteínas têm a função de controlar a homeostasia celular, protegendo o órgão contra o dano oxidativo.

O mecanismo pelo qual o treinamento físico aumenta a atividade das enzimas antioxidantes e reduz produção de ERO permanece obscuro. Todavia, concluímos que o aumento da atividade dos complexos da CTE induzido pelo treinamento físico de oito semanas pode ser o gatilho inicial para melhorar parâmetros de estresse oxidativo. Nesse sentido, futuros estudos devem ser conduzidos baseados em biologia molecular para elucidar os mecanismos responsáveis pelas adaptações nos parâmetros de estresse oxidativo.

Todos os autores declararam não haver qualquer potencial conflito de interesses referente a este artigo.
15. Navarro A, López-Cepero JM, Bández MJ, Sánchez-Pino MJ, Gómez C, Cadenas E, et al. Hippocampa mitochondrial dysfunction in rat aging. Am J Physiol Regul Integr Comp Physiol 2008;294:R501-9.

16. Chagnon P, Betard C, Robitaille Y, Cholette A, Gauvreau D. Distribution of brain cytochrome oxidase activity in various neurodegenerative diseases. Neuroreport 1995;6:711-5.

17. Mizuno Y, Ohta S, Tanaka M, Takamiya S, Suzuki K, Sato T, et al. Deficiencies in complex I subunits of the respiratory chain in Parkinson's disease. Biochem Biophys Res Commun 1989;163:1450-5.

18. Pessayre D, Abdellah M, Bernard F. Nonalcoholic steatosis and steatohepatitis. V. Mitochondrial dysfunc tion in steatohepatitis. Am J Physiol Gastrointest Liver Physiol 2002;282:G193-9.

19. Halseth AE, Fogt DL, Fregosi RF, Henriksen EJ. Metabolic responses of rat respiratory muscles to voluntary exercise training. J Appl Physiol 1995;79:902-7.

20. Bagby GJ, Johnson JL, Bennett BW, Shepherd RE. Muscle lipoprotein lipase activity in voluntarily exercising rats. J. Appl Physiol 1986;60:1623-7.

21. Fitts RH, Booth FW, Winder WW, Holloszy JO. Skeletal muscle respiratory capacity, endurance, and glycogen utilization. Am J Physiol 1975;22:1029-33.

22. Silva LA, Pinho CA, Scarabelot KS, Fraga DB, Volpato AM, Boeck CR, et al. Physical exercise increases mitochondrial function and reduces oxidative damage in skeletal muscle. Eur J Appl Physiol 2009 (in press).

23. Nakamoto H, Kaneko T, Tahara S, Hayashi E, Naito H, Radak Z, et al. Regular exercise reduces 8-oxodG in the nuclear and mitochondrial DNA and modulates the DNA repair activity in the liver of old rats. Exp Gerontol 2007;42:287-95.

24. Robinson DM, Ogilvie RW, Tullson PC, Terjung RL. Increased peak oxygen consumption of trained muscle requires increased electron flux capacity. J Appl Physiol 1994;77:1941-52.

25. Ji LL. Exercise and oxidative stress: role of the cellular antioxidant systems. Exerc Sport Sci Rev 1995;23:135-66

26. Navarro-Arevalo A, Sanchez-del-Pino MJ. Age and exercise-related changes in lipid peroxidation and superoxide dismutase activity in liver and soleus muscle tissues of rats. Mech Ageing Dev 1998; 104:91-102.

27. Venditti $\mathrm{P}$, Masullo $\mathrm{P}$, Di Meo S. Effect of training on $\mathrm{H}(2) \mathrm{O}(2)$ release by mitochondria from rat skeletal muscle. Arch Biochem Biophys 1999;372:315-20.

28. Powers SK, Criswell D, Lawler J, Ji LL, Martin D, Herb RA, et al. Influence of exercise and fiber type on antioxidant enzyme activity in rat skeletal muscle. Am J Physiol 1994;266:R375-80.

29. González B, Manso R. Induction, modification and accumulation of HSP70s in the rat liver after acute exercise: early and late responses. J Physiol 2004;556:369-85. 